\title{
On the Majorization of Analytic Functions
}

\section{A. HINKKANEN}

\section{$\S 1$. Introduction and results}

1.1. Let $G$ be an open set in the plane, and let $f$ be a function analytic in $G$ and continuous in $\bar{G}$, the closure of $G$. We say that a function $\mu$ defined for $t \geq 0$ is a majorant if $\mu$ is nonnegative, nondecreasing and continuous, and

$$
\mu(2 t) \leq 2 \mu(t)
$$

for $t \geq 0$. We consider the following majorization problem for analytic functions. Suppose that

$$
\left|f\left(z_{1}\right)-f\left(z_{2}\right)\right| \leq \mu\left(\left|z_{1}-z_{2}\right|\right)
$$

for all $z_{1}$ and $z_{2}$ on $\partial G$, the boundary of $G$. What kind of upper bound can be obtained for $\left|f\left(z_{1}\right)-f\left(z_{2}\right)\right|$ when $z_{1}, z_{2} \in \bar{G}$ ?

Suppose that $G$ has at least two finite boundary points. Gehring, Hayman and the author [3, Theorem 1] showed that if $\mu(t)=M t^{\alpha}$ where $M>0$ and $0 \leq \alpha \leq 1$, then (1.2) for all $z_{1}, z_{2} \in \partial G$ implies that (1.2) holds for all $z_{1}, z_{2} \in$ $\bar{G}$. Tamrazov [10] and the author [5, Theorem 1] showed more generally that if $\log \mu\left(e^{t}\right)$ is a concave function of $t$, then (1.2) on $\partial G$ implies (1.2) in $\bar{G}$. Tamrazov $[9$, Theorem 9.3, p. 167] had shown that the concavity condition is necessary for this result to be true. In all these cases one must assume that in any unbounded component of $G$, the function $f$ satisfies a suitable growth restriction such as $f(z)=o(|z|)$ as $z \rightarrow \infty$. For details we refer to $[3,5,10]$.

1.2. In general, the best result one can hope for is that

$$
\left|f\left(z_{1}\right)-f\left(z_{2}\right)\right| \leq C \mu\left(\left|z_{1}-z_{2}\right|\right)
$$

for $z_{1}, z_{2} \in \bar{G}$ for some constant $C>1$. Examples of Rubel, Shields and Taylor $[6$, p. 34 and Proposition 4.3, p. 38] show that one sometimes needs to take $C>1$, even if $G$ is the unit disk. So given an open set $G$, one can ask if there is an absolute constant $C$ or a constant $C$ depending on $G$ only such that (1.2) on $\partial G$ implies (1.3) in $\bar{G}$ for any $f$ and $\mu$. We assume from now on that each 
component of $G$ is bounded, to avoid problems arising from unbounded sets or functions.

If $G$ is the unit disk, one can take $C=3$ as the argument of R. Kaufman given in [6, Theorem 1.1, pp. 25-26] shows. If $G$ is a simply connected domain, one can take $C$ to be an absolute constant (for example, $C=2 \cdot 10^{7}$ ), as was shown by Rubel, Shields and Taylor $[6$, Theorem 2.7, p. 31]. Tamrazov [9, Theorem 9.1] obtained $C=108$ for simply connected domains. Next we shall explore the ideas of Tamrazov [9] more carefully. References to other earlier work on this subject can be found in [3, p. 244], and some of the earliest results can be found in $[4,8,14]$.

1.3. Let $\operatorname{cap} E$ denote the logarithmic capacity of the compact set $E$. Let $d(z, E)$ be the distance of the point $z$ from the closed set $E$. By using Tamrazov's ideas, we shall prove the following result.

Theorem 1. Let $f$ be analytic in the open set $G$, and continuous and bounded in $\bar{G}$. Suppose that $w_{1} \in \partial G, w_{2} \in G$, that there is a compact set $\Omega$ such that $w_{2}$ belongs to the unbounded component of $G \cup(\mathbf{C} \backslash \Omega)$, and that

$$
\left|z-w_{2}\right| \leq A_{1}\left|w_{1}-w_{2}\right| \quad \text { for } z \in \Omega
$$

and

$$
\operatorname{cap}((\mathbf{C} \backslash G) \cap \Omega) \geq A_{2}\left|w_{1}-w_{2}\right|
$$

for some positive constants $A_{1}$ and $A_{2}$. If (1.2) holds for $z_{1}=w_{1}$ and all $z_{2} \in \partial G$, then

$$
\left|f\left(w_{1}\right)-f\left(w_{2}\right)\right| \leq C \mu\left(\left|w_{1}-w_{2}\right|\right)
$$

where

$$
C=2 A_{1}^{2} A_{2}^{-1}\left(1+\frac{\left|w_{1}-w_{2}\right|}{d}\right)
$$

and $d=\max \left(d\left(w_{2}, \partial G\right), d\left(w_{2}, \Omega\right)\right)$.

Tamrazov essentially proved this result but considered only the case when $\Omega=E\left(w_{1}, w_{2}\right)$, where

$$
E\left(z_{1}, z_{2}\right)=\left\{\left|z-z_{1}\right| \leq \frac{1}{2}\left|z_{1}-z_{2}\right|\right\} \backslash G
$$

assuming that the conditions of Theorem 1 are satisfied for this choice of $\Omega$. So he obtained the following result [7, Theorems 4.1,6.1, 9.1], where it is assumed that $f$ is bounded and that $\partial G$ is bounded (so that $f$ remains analytic at infinity if $G$ is unbounded). 
Theorem A. If (1.2) holds for a fixed $z_{1} \in \partial G$ and all $z_{2} \in \partial G$, then

$$
\left|f\left(z_{1}\right)-f\left(z_{2}\right)\right| \leq 27 \mu\left(\left|z_{1}-z_{2}\right|\right)\left|z_{1}-z_{2}\right|\left(2 \operatorname{cap} E\left(z_{1}, z_{2}\right)\right)^{-1}
$$

for this $z_{1}$ and all $z_{2} \in \bar{G}$. Further, if for each $z_{2} \in G$, there is $z_{1} \in \partial G$ such that $\left|z_{1}-z_{2}\right|=d\left(z_{2}, \partial G\right)$ and

$$
\operatorname{cap} E\left(z_{1}, z_{2}\right) \geq \delta\left|z_{1}-z_{2}\right|
$$

where $\delta$ is a positive number depending on $G$ only, then (1.2) for all $z_{1}, z_{2} \in \partial G$ implies

$$
\left|f\left(z_{1}\right)-f\left(z_{2}\right)\right| \leq C \mu\left(\left|z_{1}-z_{2}\right|\right)
$$

for all $z_{1}, z_{2} \in \bar{G}$ with $C=\frac{9}{\delta}+2$.

If $G$ is a simply connected domain, we can take $\delta=\frac{1}{8}$ in (1.9) and $C=74$ in (1.10).

Above we have sharpened Tamrazov's arguments to obtain $C=74$ instead of his original $C=108$. For the sake of completeness, and also since Tamrazov deals with more complicated concepts in [9], we shall give a proof of Theorem 1 and derive Theorem $\mathrm{A}$ from it in $\S 2$.

1.4. In Theorems 1 and $\mathrm{A}$, the geometry of $G$ (for example, the ratios of the diameters of and the distances between the boundary components of $G$, if diameters are used to estimate capacity) has a decisive effect on how good a result we can get. The reason for this is that the proofs of Theorems 1 and A as well as the proofs of [3, Theorem 1] and [5, Theorem 1] only make use of the fact that for any $z_{0} \in \bar{G}$, the function $\log \left|f(z)-f\left(z_{0}\right)\right|$ is subharmonic for $z \in G$. This is quite sufficient in [3] and [5]. However, in general one must make better use of the fact that $f$ is a single-valued analytic function in $G$. For this purpose we shall extend the ideas of Teichmüller [11] to obtain a method based on the interplay between the Green's function and harmonic measure that will give sharper upper bounds for $\left|f\left(z_{1}\right)-f\left(z_{2}\right)\right|$. One can discuss such methods in domains whose boundary is reasonably smooth, and to facilitate this, we first note the following approximation results.

Theorem 2. Hуротнеsis: There is an absolute constant $C$ such that whenever $G$ is a bounded open set with at most two components, the boundary of $G$ consists of finitely many disjoint analytic Jordan curves, $f$ is rational with poles outside $\bar{G}$ and (1.2) holds for all $z_{1}, z_{2} \in \partial G$, then (1.3) holds for all $z_{1}, z_{2} \in \bar{G}$.

Conclusion: Whenever $G$ is open with only bounded components, $f$ is analytic in $G$ and continuous in $\bar{G}$, and (1.2) holds for all $z_{1}, z_{2} \in \partial G$, it follows that (1.3) holds for all $z_{1}, z_{2} \in \bar{G}$.

Even if there were no absolute constant $C$ as in Theorem 2, one can ask if there is such a constant depending only on the connectivity of the domain. 
Theorem 3. Let $n$ be a positive integer.

Hyротнеsis: There is a constant $C_{n}$ depending on $n$ only such that whenever $G$ is a bounded domain of connectivity $n$ whose boundary consists of $n$ disjoint analytic Jordan curves, $f$ is rational with poles outside $\bar{G}$, and (1.2) holds for all $z_{1}, z_{2} \in \partial G$, then (1.3) holds for all $z_{1}, z_{2} \in \bar{G}$ with $C$ replaced by $C_{n}$.

Conclusion: Whenever $G$ is a bounded domain of connectivity $n, f$ is analytic in $G$ and continuous in $\bar{G}$, and (1.2) holds for all $z_{1}, z_{2} \in \partial G$, it follows that (1.3) holds for all $z_{1}, z_{2} \in \bar{G}$ with $C$ replaced by $C_{n}$.

Theorem 2 will be proved in $\S 3$. The proof of Theorem 3 is similar and is omitted.

1.5. So it suffices to consider domains bounded by finitely many disjoint analytic Jordan curves. In $\S \S 4$ to 8 we develop a method for this. The main steps will be expressed in Lemmas 1 to 4 . However, we have been able to make these ideas go through completely only for doubly connected domains.

Theorem 4. If $G$ is a bounded doubly connected domain and (1.2) holds for all $z_{1}, z_{2} \in \partial G$, then (1.3) holds for all $z_{1}, z_{2} \in \bar{G}$ with $C=1.63 \cdot 10^{7}$.

Theorem 4 should be compared to the fact that if, for example, $G$ is the annulus $\{1<|z|<R\}$, then the best constant $C$ for (1.3) that we can obtain by using Theorem 1 is essentially $C=A R$ where $A$ is an absolute constant. Nevertheless, Theorem 1 remains a valuable tool by using which one can simplify situations before applying more delicate methods, and indeed deal with situations where the new methods we shall develop would not work alone. For further comments see subsection 7.5.

In $\S 8$ we reduce the problem of estimating $\left|f\left(z_{1}\right)-f\left(z_{2}\right)\right|$ to the case when $G$ is the unit disk from which finitely many Jordan domains have been deleted, such that the complement of $G$ in the unit disk has small capacity. Finally, in $\S 9$ we consider the case when $G$ is an open set with two components, and $z_{1}$ and $z_{2}$ belong to distinct components of $G$.

1.6. We recall the following lemma ([5, Lemma 2], which is an extension of [9, Lemma 4.1, p. 156]).

Lemma A. Let $G$ be an open set with at least one (finite) boundary point, let $f$ be analytic in $G$ and continuous in $\bar{G}$, and suppose that

$$
f(z)=o\left(|z|^{2}\right)
$$

as $z \rightarrow \infty$ in any unbounded component of $G$. Then for every positive $t$, we have

$$
\begin{aligned}
& \sup \left\{\left|f\left(z_{1}\right)-f\left(z_{2}\right)\right|:\left|z_{1}-z_{2}\right| \leq t, z_{1}, z_{2} \in \bar{G}\right\} \\
= & \sup \left\{\left|f\left(z_{1}\right)-f\left(z_{2}\right)\right|:\left|z_{1}-z_{2}\right| \leq t, z_{1} \in \partial G, z_{2} \in \bar{G}\right\} .
\end{aligned}
$$


Lemma A shows that to prove an estimate like (1.3) for all $z_{1}, z_{2} \in \bar{G}$, we may assume that $z_{1} \in \partial G$ and $z_{2} \in G$. If $z_{1} \in \partial G$ is fixed, one normally uses only the assumption that (1.2) holds for this $z_{1}$ and all $z_{2} \in \partial G$ to prove (1.3) for this $z_{1}$ and all $z_{2} \in \bar{G}$. In the sequel we shall take this to be understood without further comments.

\section{$\S 2$. Proof of Theorem 1}

2.1. The proof of Theorem 1 is along the lines of [9, pp. 150-155]. Let the assumptions of Theorem 1 be satisfied. Let $G_{2}$ be the component of $G$ and let $V$ be the component of $G \cup\{\infty\} \cup(\mathbf{C} \backslash \Omega)$ containing $w_{2}$, so that $w_{2} \in V, \infty \in V$, and $G_{2} \subset V$.

We define the subharmonic function $v$ in $G_{2}$ by

$$
v(z)=\log \left|f(z)-f\left(w_{1}\right)\right|-\log \mu\left(\left|w_{1}-w_{2}\right|\right) .
$$

Then $v$ is bounded above since $f$ is bounded. If $u$ is harmonic and bounded below in $G_{2}$ and $v \leq u$ on $\partial G_{2}$, then $v-u$ is bounded above in $G_{2}$ and $v \leq u$ in $G_{2}$. Hence $v\left(w_{2}\right) \leq u\left(w_{2}\right)$.

We define the harmonic function $u$ by

$$
u(z)=g\left(z, w_{2}\right)+\log \frac{2\left|z-w_{2}\right|}{\left|w_{1}-w_{2}\right|}+C_{1},
$$

where

$$
C_{1}=\log \left(1+\frac{\left|w_{1}-w_{2}\right|}{d}\right)
$$

and $g\left(z, w_{2}\right)$ is the generalized Green's function in $V$ with the pole at $z=w_{2}$. The function $g$ exists since $\operatorname{cap}(\partial V)>0$ by $(1.5)$. we have

Suppose first that $d=d\left(w_{2}, \partial G\right) \geq d\left(w_{2}, \Omega\right)$. Since $g \geq 0$ and $\partial G_{2} \subset \partial G$,

$$
u(z) \geq C_{1}+\log \frac{2 d\left(w_{2}, \partial G\right)}{\left|w_{1}-w_{2}\right|}
$$

for $z \in \partial G_{2}$. Next we estimate $v(z)$ for $z \in \partial G_{2}$. If $\left|z-w_{1}\right| \leq\left|w_{1}-w_{2}\right|$, we have $v(z) \leq 0 \leq u(z)$ by (1.2) and (2.3). Now note that by (1.1) we have

$$
\mu(L t) \leq 2 L \mu(t)
$$

for $t \geq 0$ and $L \geq 1$. So if $\left|z-w_{1}\right| \geq\left|w_{1}-w_{2}\right|$, then

$$
v(z) \leq \log \frac{\mu\left(\left|z-w_{1}\right|\right)}{\mu\left(\left|w_{1}-w_{2}\right|\right)} \leq \log \frac{2\left|z-w_{1}\right|}{\left|w_{1}-w_{2}\right|}
$$


Set $x=1+d\left|w_{1}-w_{2}\right|^{-1}>1$. One can verify that if $\left|z-w_{1}\right| \geq x\left|w_{1}-w_{2}\right|$, then

$$
\left|z-w_{1}\right| \leq x(x-1)^{-1}\left|z-w_{2}\right|
$$

while if $1<\left|z-w_{1}\right| \cdot\left|w_{1}-w_{2}\right|^{-1}<x$ (and $z \in \partial G_{2}$ ), then

$$
\frac{\left|z-w_{1}\right|}{\left|z-w_{2}\right|} \leq \frac{x\left|w_{1}-w_{2}\right|}{d\left(w_{2}, \partial G\right)}
$$

Thus in any case

$$
\log \frac{2\left|z-w_{1}\right|}{\left|w_{1}-w_{2}\right|} \leq \log \frac{2\left|z-w_{2}\right|}{\left|w_{1}-w_{2}\right|}+\log \left(1+\frac{\left|w_{1}-w_{2}\right|}{d\left(w_{2}, \partial G\right)}\right) \leq u(z) .
$$

Suppose then that $d\left(w_{2}, \partial G\right)<d\left(w_{2}, \Omega\right)=d$. Let $B$ be the disk with center $w_{2}$ and radius $d$. Then $B \subset V$, so that $h(z)=g\left(z, w_{2}\right)+\log \left|z-w_{2}\right|$ is harmonic in $B$, and $h(z) \geq \log d$ for $z \in \partial B$. Therefore $h(z) \geq \log d$ for $z \in \bar{B}$. Now one can verify that $v \leq u$ in $\partial G_{2} \cap \bar{B}$, by using (2.5) if $\left|z-w_{1}\right| \geq\left|w_{1}-w_{2}\right|$, and noting that $v(z) \leq 0$ otherwise. If $z \in \partial G_{2} \backslash \bar{B}$, one checks that $v(z) \leq u(z)$ as above, observing that $d=d\left(w_{2}, \Omega\right)$ in the definition of $x$. So in all cases $v \leq u$ on $\partial G_{2}$, and hence $v\left(w_{2}\right) \leq u\left(w_{2}\right)$.

To find an upper bound for $u\left(w_{2}\right)$, note that the harmonic function

$$
W(z)=g\left(z, w_{2}\right)-g(z, \infty)+\log \left|z-w_{2}\right|
$$

is bounded above in $V \backslash\{\infty\}$, and by (1.4) we have

$$
W(z) \leq \sup _{\zeta \in \partial V} \log \left|\zeta-w_{2}\right| \leq \log A_{1}+\log \left|w_{1}-w_{2}\right|
$$

for $z \in \partial V$. We conclude that

$$
W(z) \leq \log A_{1}+\log \left|w_{1}-w_{2}\right|
$$

for all $z \in \bar{V}$. Moreover, we have

$$
g(z, \infty)=-\log \Gamma+\int_{\partial V} \log |\zeta-z| d \nu(\zeta)
$$

where $\nu$ is the equilibrium measure of $\partial V$ (see e.g. [13, p. 83]), so that $\nu(\partial V)=$ 1 , and $\Gamma=\operatorname{cap}(\mathbf{C} \backslash V) \geq A_{2}\left|w_{1}-w_{2}\right|$ by $(1.5)$.

It follows that for $z \in V$, we have

$$
\begin{aligned}
u(z) & =W(z)+g(z, \infty)+\log \left(\frac{2}{\left|w_{1}-w_{2}\right|}\right)+C_{1} \\
& \leq g(z, \infty)+\log 2 A_{1}+C_{1} \\
& \leq \int_{\partial V} \log |\zeta-z| d \nu(\varsigma)+C_{1}+\log \frac{2 A_{1}}{A_{2}\left|w_{1}-w_{2}\right|}
\end{aligned}
$$


Since $\nu(\partial V)=1$, we obtain by the second inequality in (2.6) that

$$
v\left(w_{2}\right) \leq u\left(w_{2}\right) \leq C_{1}+\log 2 A_{1}^{2} A_{2}^{-1} .
$$

This yields (1.6) with $C$ given by (1.7). Theorem 1 is proved.

2.2. To deduce Theorem A from Theorem 1 , note that if

$$
\Omega=\left\{\left|z-w_{1}\right| \leq \frac{1}{2}\left|w_{1}-w_{2}\right|\right\}
$$

we can use Theorem 1 with $A_{1}=\frac{3}{2}$ and $A_{2}=\left|w_{1}-w_{2}\right|^{-1}$ cap $E\left(w_{1}, w_{2}\right)$. Then $d\left(w_{2}, \Omega\right)=\frac{1}{2}\left|w_{1}-w_{2}\right| \leq d$, so that $C A_{2} \leq \frac{27}{2}$. This gives (1.8). Suppose then that (1.9) holds as stated in Theorem A. Let $w_{1}$ and $w_{2}$ be as above, with $\left|w_{1}-w_{2}\right|=d\left(w_{2}, \partial G\right)$. Then $A_{2} \geq \delta$ and $d \geq\left|w_{1}-w_{2}\right|$, so that (1.6) holds with $C=\frac{9}{\delta}$. If $w_{3} \in \partial G$, then

$$
\left|w_{3}-w_{1}\right| \leq\left|w_{3}-w_{2}\right|+\left|w_{2}-w_{1}\right| \leq 2\left|w_{3}-w_{2}\right|
$$

since $\left|w_{1}-w_{2}\right|=d\left(w_{2}, \partial G\right) \leq\left|w_{3}-w_{2}\right|$, and by (1.2), (1.1), and (1.7),

$$
\begin{aligned}
\left|f\left(w_{3}\right)-f\left(w_{2}\right)\right| & \leq\left|f\left(w_{3}\right)-f\left(w_{1}\right)\right|+\left|f\left(w_{1}\right)-f\left(w_{2}\right)\right| \\
& \leq \mu\left(\left|w_{3}-w_{1}\right|\right)+\frac{9 \mu\left|w_{1}-w_{2}\right|}{\delta} \\
& \leq\left(2+\frac{9}{\delta}\right) \mu\left(\left|w_{3}-w_{2}\right|\right),
\end{aligned}
$$

which proves (1.10).

If $G$ is a simply connected domain not containing the point at infinity and if $z_{1} \in \partial G, z_{2} \in G$, then it is impossible to have

$$
\partial G \subset\left\{\left|z-z_{1}\right| \leq \frac{1}{2}\left|z_{1}-z_{2}\right|\right\}=D_{1}
$$

say. Hence $\partial G$ contains a continuum $\gamma$ joining $z_{1}$ in $D_{1}$ to a point on $\partial D_{1}$. The diameter of $\gamma$ is at least $\frac{1}{2}\left|z_{1}-z_{2}\right|$, and the capacity of $\gamma$ is at least one quarter of this (see e.g. [1, p. 29]). Therefore we can take $\delta=\frac{1}{8}$ in (1.9) and hence $C=74$ in (1.10).

We note that if $G$ is a simply connected subdomain of the extended complex plane $\overline{\mathbf{C}}$ and contains a neighborhood of infinity, and if $f$ is bounded, then Tamrazov's argument [9, p. 165] shows that we can still take $C=74$ in (1.10). Theorem A is proved. 


\section{$\S 3$. Proof of Theorem 2}

3.1. Let the assumptions of Theorem 2 be satisfied, and let $G$ be an open set with only bounded components. Let $f$ be analytic in $G$ and continuous in $\bar{G}$. Let $\mu$ be a majorant satisfying (1.1), and suppose that (1.2) holds for all $z_{1}, z_{2} \in \partial G$. We need to show that

$$
\left|f\left(z_{1}\right)-f\left(z_{2}\right)\right| \leq C \mu\left(\left|z_{1}-z_{2}\right|\right)
$$

for all $z_{1}, z_{2} \in \bar{G}$.

Clearly it suffices to prove (3.1) when $z_{1}, z_{2} \in G$. Then (3.1) follows for $z_{1}, z_{2} \in \bar{G}$ by continuity. Hence choose $z_{1}, z_{2} \in G$ and let $D_{i}$ be the component of $G$ containing $z_{i}$ for $i=1,2$.

Let $S(\delta)$ be the grid in the plane determined by all squares whose vertices are of the form $(m \delta, n \delta)$ for integers $m$ and $n$ and that have side $\delta$.

Let $\varepsilon>0$ be given, and let $M$ be the diameter of $D=D_{1} \cup D_{2}$. Since $\mu$ is uniformly continuous on any bounded interval, we may choose $\delta \in(0,1)$ so small that

$$
\mu(t+6 \delta) \leq \mu(t)+\varepsilon
$$

for $0 \leq t<t+6 \delta \leq M+6$. Since $f$ is uniformly continuous on the compact set $\bar{D}$, we can take $\delta$ so small that

$$
|f(z)-f(w)| \leq \varepsilon
$$

whenever $z, w \in \bar{D}$ and $|z-w| \leq 3 \delta$. Finally, we take $\delta$ so small that

$$
\min _{i=1,2} \operatorname{dist}\left(z_{i}, \partial D_{i}\right) \geq 3 \sqrt{2} \delta
$$

3.2. Let $\delta$ be chosen so that it satisfies all of the conditions above, and let the open set $V$ be the union of all those open squares of $S(\delta)$ that are contained in $D$. Set

$$
U=\text { interior } \bar{V}
$$

so that $U \subset \bar{G}$ and $U=\operatorname{int} \bar{U}$. Since $f$ is analytic in $G$, continuous in $\bar{G}$, and since $U \backslash G$ has finite linear measure, it follows that $f$ is analytic in $U$ and continuous in $\bar{U}[2$, Theorem 2.3, p. 66].

Clearly $U$ has only finitely many components, and any component $W$ is bounded by finitely many closed curves. Since $U=$ int $\bar{U}$, any such curve $\gamma \subset \partial W$ can intersect itself at only finitely many points. Let $W_{1}$ be the component of the complement of $\gamma$ in the extended plane containing $W$ (but not necessarily all of $U)$. Let $\varphi$ be a conformal mapping of the unit disk onto the simply connected domain $W_{1}$. Set $\Gamma=\varphi(|z|=r)$ where $0<r<1$. Choosing $r$ close enough to 
one and going through all components $W$ and all curves $\gamma$, we obtain a finite set of disjoint analytic Jordan curves $\Gamma$ which form the boundary of an open set $\Omega$ such that $\bar{\Omega} \subset U$. Further we may assume that for any $z \in \partial \Omega$ there is $w \in \partial U$ such that $|z-w| \leq \delta$.

Suppose that $a_{1}, a_{2} \in \partial \Omega$. Then there are $b_{1}, b_{2} \in \partial U$ with $\left|a_{i}-b_{i}\right| \leq \delta$. Now $b_{1}$ is contained in a closed line segment $\sigma$ belonging to $\partial U$. Also $\sigma \subset \partial S$ for some closed square $S$ in $S(\delta)$ such that $S \cap \partial G \neq \varnothing$. So we see that there are $c_{1}, c_{2} \in \partial G$ such that $\left|b_{i}-c_{i}\right| \leq \sqrt{2} \delta<2 \delta$. Hence $\left|a_{i}-c_{i}\right|<3 \delta$.

By (3.3) and (1.2) we have

$$
\begin{aligned}
\left|f\left(a_{1}\right)-f\left(a_{2}\right)\right| & \leq\left|f\left(a_{1}\right)-f\left(c_{1}\right)\right|+\left|f\left(c_{1}\right)-f\left(c_{2}\right)\right|+\left|f\left(c_{2}\right)-f\left(a_{2}\right)\right| \\
& \leq 2 \varepsilon+\mu\left(\left|c_{1}-c_{2}\right|\right) .
\end{aligned}
$$

Since $\left|c_{1}-c_{2}\right| \leq 6 \delta+\left|a_{1}-a_{2}\right|,(3.2)$ gives $\left|f\left(a_{1}\right)-f\left(a_{2}\right)\right| \leq \mu\left(\left|a_{1}-a_{2}\right|\right)+3 \varepsilon$.

3.3. Since $\mathbf{R}^{2} \backslash \bar{\Omega}$ has finitely many components, it follows from an extension of Mergelyan's theorem [7, p. 427] (or from Runge's theorem applied to a neighborhood of $\bar{\Omega}$ ) that there is a rational function $g$ with poles outside $\bar{\Omega}$ such that $|f(z)-g(z)| \leq \varepsilon$ for $z \in \bar{\Omega}$. Hence

$$
\left|g\left(a_{1}\right)-g\left(a_{2}\right)\right| \leq \mu\left(\left|a_{1}-a_{2}\right|\right)+5 \varepsilon=\nu\left(\left|a_{1}-a_{2}\right|\right)
$$

for all $a_{1}, a_{2} \in \partial \Omega$, where $\nu(t)=\mu(t)+5 \varepsilon$ is a majorant satisfying (1.1).

Applying the assumption of Theorem 2 to any two components of $\Omega$ separately, we see that

$$
\left|g\left(a_{1}\right)-g\left(a_{2}\right)\right| \leq C \nu\left(\left|a_{1}-a_{2}\right|\right)
$$

and hence

$$
\left|f\left(a_{1}\right)-f\left(a_{2}\right)\right| \leq C \mu\left(\left|a_{1}-a_{2}\right|\right)+(5 C+2) \varepsilon
$$

for all $a_{1}, a_{2} \in \bar{\Omega}$. By (3.4) and the construction of $\Omega$ we have $z_{1}, z_{2} \in \bar{\Omega}$. Namely, (3.4) guarantees that if $z_{1}$, say, belongs to a closed square $S_{1}$ in the grid $S(\delta)$ and if the eight closed squares immediately surrounding $S_{1}$ are $S_{2}, \ldots, S_{9}$, then int $\bigcup_{i=1}^{9} S_{i} \subset U$. Hence $\partial \Omega$ cannot intersect int $S_{1}$. So int $S_{1} \subset \Omega$, and $z_{1} \in S_{1} \subset \bar{\Omega}$. Since $\varepsilon$ is arbitrary, we obtain (3.1). This proves Theorem 2 .

\section{$\S 4$. The set-up in domains bounded by analytic curves}

4.1. Let $G$ be a bounded domain of connectivity $N+1$, whose boundary consists of the $N+1$ disjoint analytic Jordan curves $\Gamma_{i}$, where $0 \leq i \leq N$, $\Gamma_{0}$ being the outer contour. Suppose that $z_{1} \notin G$ and assume, without loss of generality, that $z_{1}=0$. Choose $z_{0} \in G$ and let $g(z)=g\left(z, z_{0}\right)$ be the Green's function of $G$ with the pole at $z=z_{0}$. Let $\omega_{i}(z)$ be the harmonic measure of $\Gamma_{i}$ for $0 \leq i \leq N$. Suppose that $f$ is analytic in a neighborhood of $\bar{G}$, and assume 
that $|f(z)| \leq \mu(|z|)$ for $z \in \partial G$. So $f(0)=0$ if $z_{1}=0 \in \partial G$ and $\mu(0)=0$. Note that $f$ has only finitely many zeros in $\bar{G}$, say $a_{i}$ for $1 \leq i \leq \kappa$, with due count of multiplicity. We assume that $a_{i} \in G$ for $1 \leq i \leq \bar{K}$ and that $a_{i} \in \partial G$ for $K+1 \leq i \leq \kappa$.

We write $M(t)=\log \mu\left(e^{t}\right)$. Because suitable approximation arguments are available, we could assume without loss of generality that $M(t)$ is twice continuously differentiable. Indeed, if $\mu$ is a majorant and $\varepsilon>0$, we can find a majorant $\nu(t)$ such that

$$
\mu(t) \leq \nu(t) \leq \mu(t)+\varepsilon \quad \text { for all } t \geq 0
$$

and such that $\log \nu\left(e^{t}\right)$ is twice continuously differentiable. If

$$
\left|f\left(z_{1}\right)-f\left(z_{2}\right)\right| \leq \mu\left(\left|z_{1}-z_{2}\right|\right) \leq \nu\left(\left|z_{1}-z_{2}\right|\right)
$$

for all suitable $z_{1}$ and $z_{2}$, we deduce that

$$
\left|f\left(z_{1}\right)-f\left(z_{2}\right)\right| \leq C \nu\left(\left|z_{1}-z_{2}\right|\right) \leq C \mu\left(\left|z_{1}-z_{2}\right|\right)+C \varepsilon
$$

for all relevant $z_{1}$ and $z_{2}$. Since $\varepsilon$ is arbitrary, we obtain (1.3).

However, at one point in the proof of Theorem 4 in subsection 7.3 , the clearest way to use the ideas involved is to make the function $M_{1}(t)$ there piecewise linear without any modifications. If $M(t)$ is piecewise linear, we can interpret $M^{\prime \prime}(t)$ as a linear combination of one-dimensional Dirac delta-distributions, which can be used in integration arguments. As there is no mathematical problem about doing this, we feel that it is justified to reserve the possibility of making $M(t)$ piecewise linear whenever convenient.

We set $u(z)=\log \left[\frac{\mu(|z|)}{|f(z)|}\right]$ for $z \in \partial G$. Hence $u(z) \geq 0$. The next lemma contains the basic formalism that we shall use.

Lemma 1. The expression

$$
\begin{aligned}
N_{j}=\frac{1}{2 \pi} \int_{\partial G} M(\log r) \frac{\partial \omega_{j}}{\partial n} d s & \\
& -\frac{1}{2 \pi} \int_{\partial G} u(z) \frac{\partial \omega_{j}}{\partial n} d s+\sum_{i=1}^{K} \omega_{j}\left(a_{i}\right)
\end{aligned}
$$

is an integer for $0 \leq j \leq N$. We can write

$$
\begin{aligned}
-N_{j}=\frac{1}{2 \pi} \int_{\operatorname{int} \Gamma_{0}} & r^{-1} M^{\prime \prime}(\log r) \omega_{j}(z) d r d \vartheta \\
& +\frac{1}{2 \pi} \int_{\partial G} u(z) \frac{\partial \omega_{j}}{\partial n} d s-\sum_{i=1}^{K} \omega_{j}\left(a_{i}\right),
\end{aligned}
$$

where, in the integral over int $\Gamma_{0}, \omega_{0}(z)$ is to be replaced by $\omega_{0}(z)-1$ if $j=0$. 
Further, we have

$$
\begin{aligned}
\log \frac{\left|f\left(z_{0}\right)\right|}{\mu\left(\left|z_{0}\right|\right)}=\frac{1}{2 \pi} \int_{G} r^{-1} M^{\prime \prime}(\log r) g(z) d r d \vartheta \\
+\frac{1}{2 \pi} \int_{\partial G} u(z) \frac{\partial g}{\partial n} d s-\sum_{i=1}^{K} g\left(a_{i}\right) .
\end{aligned}
$$

Here $z=r e^{i \vartheta}, d s$ is the arc length along $\partial G$, and $\frac{\partial}{\partial n}$ denotes the derivative in the direction of the outer normal vector to $\partial G$. Of course, int $\Gamma_{0}$ is the interior of the curve $\Gamma_{0}$, which is a Jordan domain containing $G$. It is understood that $g=0$ outside $G$ and that in each component $D$ of the complement of $G$, any $\omega_{j}$ is equal to its constant value, zero or one, on $\partial D$. (When calculating the normal derivatives of $g$ and $\omega_{j}$, we take into account only the values of these functions in $G$.) Observe also the formal similarity between (4.2) and (4.3): if we replace $\omega_{j}$ by $g$ in (4.2), we get (4.3).

If $\log \mu\left(e^{t}\right)$ is concave, then $M^{\prime \prime}(t) \leq 0$ for all $t$. Since $\frac{\partial g}{\partial n} \leq 0$ on $\partial G$, the right-hand side of (4.3) is then $\leq 0$ and so $\left|f\left(z_{0}\right)\right| \leq \mu\left(\left|z_{0}\right|\right)$. Hence we recover [5, Theorem 1] in this case.

4.2. Before proceeding any further, we make some remarks concerning how Lemma 1 corresponds to Teichmüller's results in [11], and how our work will develop after the proof of Lemma 1. Let us denote the right-hand side of (4.3) by $I_{1}+I_{2}+I_{3}$. The term $I_{1}$ can be positive or negative, and it could be large and positive. The terms $\mathrm{I}_{2}$ and $\mathrm{I}_{3}$ are always nonpositive, as we observed above.

In [11], Teichmüller considers a function $f$ analytic in an annulus $A=$ $\{1<|z|<R\}$ and assumes that $|f(z)| \leq 1$ for $|z|=1$ and $|f(z)| \leq L$ for $|z|=R$. Therefore he essentially defines $u(z)=\log L-\log |f(z)|$ for $|z|=R$ and $u(z)=-\log |f(z)|$ for $|z|=1$ so that $u(z) \geq 0$ on $\partial A$, and obtains a formula for $\left|f\left(z_{0}\right)\right|$ when $z_{0} \in A$ involving terms such as $\mathrm{I}_{2}$ and $\mathrm{I}_{3}$ above. (His set-up is such that the term corresponding to $I_{1}$ looks somewhat different.) However, his methods can be used equally well even if we do not majorize the maximum modulus of $f$, that is, majorize $|f(z)|$ by a function that takes constant values on circles or more generally on the boundary components of the domain, but majorize $|f(z)|$ by a function such as $\mu(|z|)$ that varies with $|z|$. Indeed, any function of $z$ (instead of a quantity depending on $|z|$ alone) could be used here.

The fact that the right-hand side of (4.1) is an integer is equivalent to saying that if $z$ goes around the curve $\Gamma_{j}$, then $f(z)$ traces a closed curve, and so the change of $\arg f(z)$ is an integral multiple of $2 \pi$. This is essentially what it means for $f$ to be single-valued in a domain that may be multiply connected. Of course, if $\Gamma_{j}$ contains a zero of $f$, then the variation of the argument of $f(z)$ might be half an integer times $2 \pi$. This could happen for at least the component of $\partial G$ containing $z_{1}=0$ since $f(0)=0$ by our assumption. However, the proof of (4.1) takes this into account, and the expressions on the right-hand side of (4.1) and (4.2) will always be integers. 
That the right-hand side of (4.1) is an integer for all $j$ essentially characterizes the assumption that $f$ is single-valued. Adding the equations (4.1) for $0 \leq j \leq N$ and taking into account that the sum of the harmonic measures $\omega_{j}$ for $0 \leq j \leq N$ is identically the constant one, we obtain

$$
\sum_{j=0}^{N} N_{j}=K \geq 0,
$$

where $K$ is the number of zeros of $f$ in $G$, with due count of multiplicity. On the other hand, there are examples of single-valued functions $f$ for which the $N_{j}$ could be arbitrarily prescribed integers (positive, negative or zero) only subject to (4.4). To see this, we merely need to take $f$ to be a rational function with no poles in $\bar{G}$, and arrange so that for each $j$, the function $f$ has a suitable number of zeros and poles in the component of the complement of $G$ bounded by $\Gamma_{j}$. The number of these zeros and poles together with the number of extra zeros of $f$ on $\Gamma_{j}$, if any, determines the winding number of $f$ along $\Gamma_{j}$ around the origin, which essentially gives $N_{j}$. In this case, (4.4) reflects the fact that $f$ must have an equal number of zeros and poles in the extended complex plane, with due count of multiplicity.

4.3. The next question is whether the terms $I_{2}$ and $I_{3}$ on the right-hand side of (4.3) can cancel a substantial part of $\mathrm{I}_{1}$. If $u \equiv 0$ on $\partial G$ and $f$ has no zeros in $G$, then $\mathrm{I}_{2}=\mathrm{I}_{3}=0$. But if this is the case, then the first term on the right-hand side of (4.2) must be an integer for $0 \leq j \leq N$, which could presumably happen only under exceptional circumstances. Next if all the zeros of $f$ are close to $\partial G$ so that $\left|\mathrm{I}_{3}\right|$ is small, then the third term on the right-hand side of (4.2) is perhaps not an integer but close to one. Hence it may be necessary for $u(z)$ to be sometimes large to provide the required adjustment and this could make $\left|\mathrm{I}_{2}\right|$ large.

But we do not really know exactly where the zeros of $f$ are and how large $u$ is or how $u$ might vary. We ask how we could make use of $\mathrm{I}_{2}$ and $\mathrm{I}_{3}$ on the basis of the knowledge that certain quantities are integers. The great idea of Teichmüller was that one possible way of doing this is to construct a real-valued function $h$ on the real axis, periodic with period one, which is in some way related to the problem. Then any integer will be a period of $h$. This, together with a purely real analytic lemma of Teichmüller (Lemma $B$ in subsection 6.3 below), allows us to replace $\mathrm{I}_{2}+\mathrm{I}_{3}$ by a single term $-h(\gamma)$, where $\gamma$ involves the mean values of harmonic measure over circles centered at the origin while $h$ gives the minimum value of a Green's function along a level curve of harmonic measure.

To be able to use this method, certain other technicalities must be taken care of. It was not difficult for Teichmüller to do this in an annulus, since there the level curves of the harmonic measure of the outer (or inner) contour are concentric circles, and if the pole of the Green's function $g$ is positive, for 
example, then the minimum of $g$ on each circle is attained on the negative axis. So many questions can be studied explicitly there. However, everything that is required for the method to apply in the formal sense at least, is valid in finitely connected domains, and this is essentially proved in Lemmas 2 and 3 in $\S 6$ below.

Of course, we may lose something when squeezing $I_{1}+I_{3}$ to a single term $-h(\gamma)$. The term $I_{3}$ may contain Green's functions at several points while $h$ only gives the minimum of $g$ on some curve. Thus it is necessary to choose $h$ in a judicious way, and out of the functions $h_{j}$ defined by (6.1) below we could choose one depending on $z_{0}$ and $\mu$. When $G$ is doubly connected, we shall not lose too much, but in a domain of higher connectivity some other choice for $h$ might be called for. Therefore another suggestion for $h$ will be mentioned in subsection 7.5.

Using only the fact that $\log |f|$ is subharmonic amounts to ignoring $\mathrm{I}_{2}$ and $I_{3}$ and estimating only $I_{1}$, even though the earlier authors naturally did not express the matter exactly in this way. This can lead to the best result only when $G$ is simply connected. For then we can prescribe $\log |f(z)|$ to be equal to $\log \mu(|z|)$ on $\partial G$, extend $\log |f|$ to a harmonic function $U$ in $G$, and find a single--valued harmonic conjugate $V$ for $U$ in $G$. Then we set $f=\exp (U+i V)$ so that $f(z) \neq 0$ for $z \in G$. Also $u \equiv 0$ on $\partial G$, and so $\mathrm{I}_{2}=\mathrm{I}_{3}=0$.

Estimating $\mathrm{I}_{1}-h(\gamma)$ in an annulus essentially amounts to showing that the mean value of the Green's function $g$ over a circle does not exceed an absolute constant plus the minimum of $g$ over a level curve which now happens to be the same circle. Because of the convexity properties of the means of $g$, it suffices to check this when the circle contains the pole of $g$. The calculations related to this will be performed in subsection 7.4. Teichmüller did not try to prove that some quantity is less than an absolute constant so that he did not deal with such estimates.

\section{$\S 5$. Proof of Lemma 1}

5.1. Let the notation be as in subsection 4.1 , and let the situation be as in Lemma 1. We shall apply Green's formula

$$
\int_{G}(u \Delta v-v \Delta u) d x d y=\int_{\partial G}\left(u \frac{\partial v}{\partial n}-v \frac{\partial u}{\partial n}\right) d s .
$$

We may apply (5.1) also to suitable distributions. For example, $\Delta \log |z|=$ $2 \pi \delta(z)$

$$
\Delta \log |f(z)|=2 \pi \sum_{i=1}^{\kappa} \delta\left(z-a_{i}\right)
$$

and $\Delta g\left(z, z_{0}\right)=-2 \pi \delta\left(z-z_{0}\right)$, where $\delta(z)$ is the two-dimensional Dirac deltadistribution. 
Taking $u=\log |f|$ and $v=\omega_{j}$ in (5.1), we get

$$
\frac{1}{2 \pi} \int_{\partial G} \log |f| \frac{\partial w_{j}}{\partial n} d s+\sum_{i=1}^{\kappa} \varepsilon_{i j} \omega_{j}\left(a_{i}\right)=\frac{1}{2 \pi} \int_{\Gamma_{j}} \frac{\partial \log |f|}{\partial n} d s
$$

where $\varepsilon_{i j}=\frac{1}{2}$ if $a_{i} \in \Gamma_{j}$ and $\varepsilon_{i j}=1$ otherwise. Now

$$
\frac{1}{2 \pi} \int_{\Gamma_{j}} \frac{\partial \log |f|}{\partial n} d s=\frac{1}{2 \pi} \int_{\Gamma_{j}} d \arg f=\ell_{j}+\sum_{i=1}^{\kappa}\left(1-\varepsilon_{i j}\right)
$$

for some integer $\ell_{j}$. Since $\log |f(z)|=M(\log |z|)-u(z)$, we obtain (4.1).

Next take $u=\log |f|$ and $v=g=g\left(z, z_{0}\right)$ in (5.1). This gives

$$
\begin{aligned}
& \log \left|f\left(z_{0}\right)\right|=-\frac{1}{2 \pi} \int_{\partial G} \log |f| \frac{\partial g}{\partial n} d s-\sum_{i=1}^{\kappa} g\left(a_{i}\right) \\
&=-\frac{1}{2 \pi} \int_{\partial G} M(\log |z|) \frac{\partial g}{\partial n} d s+\frac{1}{2 \pi} \int_{\partial G} u(z) \frac{\partial g}{\partial n} d s \\
&-\sum_{i=1}^{\kappa} g\left(a_{i}\right) .
\end{aligned}
$$

If $M(t)$ is twice continuously differentiable, then

$$
\Delta M(\log |z|)=|z|^{-2} M^{\prime \prime}(\log |z|)
$$

Using (5.1) with $u=g$ and $v=M(\log |z|)$, we get

$$
-\frac{1}{2 \pi} \int_{\partial G} M(\log |z|) \frac{\partial g}{\partial n} d s=\log \mu\left(\left|z_{0}\right|\right)+\frac{1}{2 \pi} \int_{G}|z|^{-2} g M^{\prime \prime}(\log |z|) d x d y .
$$

This gives (4.3). The same applies if $M(t)$ is piecewise linear and $M^{\prime \prime}(t)$ is interpreted in terms of Dirac's delta-distribution on the real axis. All the integrals exist since (1.1) implies that $|M(\log |z|)| \leq|\log | z||+$ constant as $z \rightarrow 0$.

5.2. To prove $(4.2)$, we apply $(5.1)$ to $u=\omega_{j}$ and $v=M(\log |z|)$ to obtain

$$
\begin{gathered}
-\frac{1}{2 \pi} \int_{\partial G} M(\log |z|) \frac{\partial \omega_{j}}{\partial n} d s=\frac{1}{2 \pi} \int_{G}|z|^{-2} \omega_{j}(z) M^{\prime \prime}(\log |z|) d x d y \\
-\frac{1}{2 \pi} \int_{\Gamma_{j}} \frac{\partial M(\log |z|)}{\partial n} d s
\end{gathered}
$$


Now (5.1) with $u=M(\log |z|)$ and $v=1$, applied to int $\Gamma_{j}$ instead of $G$, gives

$$
-\frac{1}{2 \pi} \int_{\Gamma_{j}} \frac{\partial M(\log |z|)}{\partial n} d s=\frac{s}{2 \pi} \int_{\operatorname{int} \Gamma_{j}}|z|^{-2} M^{\prime \prime}(\log |z|) d x d y
$$

where $s=-1$ if $j=0$ and $s=1$ for $1 \leq j \leq N$. The reason for this difference is that the outer normal to $\partial G$ along $\Gamma_{j}$ is the inner normal to $\partial$ (int $\Gamma_{j}$ ) if $1 \leq j \leq N$. Combining these results and taking into account how the functions $\omega_{j}$ were extended to the plane, we obtain (4.2), and Lemma 1 is proved.

\section{$\S 6$. Extension of Teichmüller's method}

6.1. In this section we obtain an upper bound for the right-hand side of (4.3) making use of the fact that the $N_{j}$ are integers. We shall extend the idea of Teichmüller that he used to obtain the sharp form of Hadamard's three circles theorem ([11]; see also [1, pp. 45-47]). Following Teichmüller, we set

$$
h_{j}(t)=\min \left\{g\left(z, z_{0}\right): \omega_{j}(z)=t\right\}
$$

for $0 \leq t \leq 1$, so that $h_{j}(0)=h_{j}(1)=0$. One can check that $h_{j}$ is continuous on $[0,1]$ and that the one-sided derivatives $h_{j}^{\prime}(0+)$ and $h_{j}^{\prime}(1-)$ are finite. Since $\omega_{j}$ is harmonic and $g$ is superharmonic, it follows that $h_{j}$ is concave on $[0,1]$. For the sake of completeness, we give the simple proof of this fact.

Lemma 2. Let $u$ be subharmonic and bounded above and let $v$ be harmonic in a domain D. Suppose that the interval $(a, b) \subset v(D)$ and that the closure of $v^{-1}([c, d])$ is contained in $D$ whenever $a<c \leq d<b$. Then

$$
h(t)=\sup \{u(z): z \in D \text { and } v(z)=t\}
$$

is convex on $(a, b)$.

Applying Lemma 2 to $D=G, u=-g, v=\omega_{j}, h=-h_{j}$ and $a=0, b=1$, we see that $h_{j}$ is concave on $(0,1)$ and hence, by continuity, on $[0,1]$.

To prove Lemma 2, suppose that $a<c<d<b$, set $V=\{z \in D$ : $c<v(z)<d\}$, so that $\bar{V} \subset D$, and let $L(t)$ be the linear function on $[c, d]$ with $L(c)=h(c), L(d)=h(d)$. We have to prove that $h(t) \leq L(t)$ for $c<t<d$. But $w(z)=u(z)-L(v(z))$ is subharmonic and bounded above in $V$. Also $w \leq 0$ on $\partial V \subset D$, since $z \in \partial V$ implies that $v(z)=c$ or $v(z)=d$. If $c<t<d$ and $\varepsilon>0$, there is $z \in V$ such that $v(z)=t$ and $u(z)>h(t)-\varepsilon$. Hence

$$
h(t)-L(t)-\varepsilon<u(z)-L(t)=w(z) \leq 0,
$$

and since $\varepsilon$ is arbitrary, we conclude that $h(t) \leq L(t)$. This proves Lemma 2 . 
6.2. To get a connection between the right-hand sides of (4.2) and (4.3), we need the following lemma.

Lemma 3. For $0 \leq j \leq N$, we have

$$
\frac{\partial g}{\partial n} \leq h_{j}^{\prime}(1-) \frac{\partial \omega_{j}}{\partial n} \quad \text { on } \Gamma_{j}
$$

and

$$
\frac{\partial g}{\partial n} \leq h_{j}^{\prime}(0+) \frac{\partial \omega_{j}}{\partial n} \quad \text { on } \Gamma_{i} \text { for } i \neq j
$$

Note that $\frac{\partial g}{\partial n}<0$ on $\partial G$, while $\frac{\partial \omega_{j}}{\partial n}>0$ on $\Gamma_{j}$ and $\frac{\partial \omega_{j}}{\partial n}<0$ on $\Gamma_{i}$ if $i \neq j$. Also, $h_{j}^{\prime}(0+)>0$ and $h_{j}^{\prime}(1-)<0$.

We only prove (6.2). Suppose that $w \in \Gamma_{j}$. Without loss of generality we assume that $w=0$, that the segment $(-\varepsilon, 0)$ is contained in $G$ for some positive $\varepsilon$, and that the outer normal vector to $\partial G$ at the origin points in the direction of the positive real axis. Then at the origin, we have

$$
\begin{aligned}
\frac{\partial g}{\partial n} & =\lim _{\delta \downarrow 0} \frac{g(-\delta)}{-\delta} \leq \lim _{\delta \downarrow 0} \frac{h_{j}\left(\omega_{j}(-\delta)\right)}{\omega_{j}(-\delta)-1} \frac{\omega_{j}(-\delta)-1}{-\delta} \\
& =h_{j}^{\prime}(1-) \frac{\partial \omega_{j}}{\partial n}
\end{aligned}
$$

since $g(-\delta) \geq h_{j}\left(\omega_{j}(-\delta)\right)$ and $\omega_{j}(-\delta) \rightarrow 1$ as $\delta \downarrow 0$. This proves (6.2), and the proof of (6.3) is similar. So Lemma 3 is true.

6.3. We extend $h_{j}$ to be a periodic function of period one on the real axis. For such a function, Teichmüller proved the following result.

Lemma $\boldsymbol{B}$ ([11, p. 17], [12, p. 277], see also [1, Lemma 3.2, p. 47]). Let $h$ be continuous and concave on $[0,1]$ with $h(0)=h(1)=0$, and suppose that $h(t+1)=h(t)$ for all real $t$. Then

$$
h\left(A_{1}-A_{2}+\sum_{i=1}^{K} t_{i}\right) \leq A_{1} h^{\prime}(0+)-A_{2} h^{\prime}(1-)+\sum_{i=1}^{K} h\left(t_{i}\right)
$$

whenever $A_{1} \geq 0, A_{2} \geq 0$, and the $t_{i}$ are real.

Using the notation of $\S 4$, we set $t_{i j}=\omega_{j}\left(a_{i}\right)$ and note that then

$$
-g\left(a_{i}\right) \leq-h_{j}\left(t_{i j}\right)
$$


By (4.3) and Lemma 3 we get, with $z=r e^{i \vartheta}$,

$$
\begin{aligned}
\log \frac{\left|f\left(z_{0}\right)\right|}{\mu\left(\left|z_{0}\right|\right)} \leq \frac{1}{2 \pi} \int_{G} & r^{-1} M^{\prime \prime}(\log r) g(z) d r d \vartheta \\
& +\frac{h_{j}^{\prime}(1-)}{2 \pi} \int_{\Gamma_{j}} u(z) \frac{\partial \omega_{j}}{\partial n} d s \\
& +\frac{h_{j}^{\prime}(0+)}{2 \pi} \sum_{i \neq j} \int_{\Gamma_{i}} u(z) \frac{\partial \omega_{j}}{\partial n} d s-\sum_{i=1}^{K} h_{j}\left(t_{i j}\right) .
\end{aligned}
$$

Now we apply Lemma B to $h=h_{j}$, taking the $t_{i}$ to be the numbers $t_{i j}$ for $1 \leq i \leq K$, and taking

$$
A_{1}=-\frac{1}{2 \pi} \sum_{i \neq j} \int_{\Gamma_{i}} u(z) \frac{\partial \omega_{j}}{\partial n} d s, \quad A_{2}=\frac{1}{2 \pi} \int_{\Gamma_{j}} u(z) \frac{\partial \omega_{j}}{\partial n} d s .
$$

Then, in view of $(6.6)$, we get

$$
\log \frac{\left|f\left(z_{0}\right)\right|}{\mu\left(\left|z_{0}\right|\right)} \leq \frac{1}{2 \pi} \int_{G} r^{-1} M^{\prime \prime}(\log r) g(z) d r d \vartheta-h_{j}\left(\gamma_{j}\right)
$$

where

$$
\gamma_{j}=\sum_{i=1}^{K} \omega_{j}\left(a_{i}\right)+A_{1}-A_{2} .
$$

But we can replace $\gamma_{j}$ by $\gamma_{j}+n$ for any integer $n$. Hence by (4.2) we may take

$$
\gamma_{j}=\frac{1}{2 \pi} \int_{\operatorname{int} \Gamma_{0}} r^{-1} M^{\prime \prime}(\log r) \omega_{j}(z) d r d \vartheta
$$

where $\omega_{0}(z)$ is to be replaced by $\omega_{0}(z)-1$ if $j=0$.

We need to find an upper bound for the right-hand side of (6.7) for some $j$ that may be allowed to vary with $z_{0}$ and $M(t)$. When applying Lemma $\mathrm{B}$, we have lost some information, and it is not clear whether or not this is too crude in general. However, we can use (6.7) and (6.8) to prove Theorem 4.

\section{$\S 7$ Proof of Theorem 4}

7.1. To prove Theorem 4 , it seems best to use Tamrazov's methods first to reduce the problem to the situation when the doubly connected domain is an annulus. This will considerably simplify the estimation of (6.7).

Lemma 4. Let $G$ be as in $\S 4$, with $z_{1}=0 \notin G$. Suppose that $|f(z)| \leq \mu(|z|)$ for $z \in \partial G$. If $0 \in \Gamma_{0}$, then

$$
\left|f\left(z_{0}\right)\right| \leq 74 \mu\left(\left|z_{0}\right|\right) \quad \text { for } z_{0} \in \bar{G} .
$$

If $0 \in\left(\right.$ int $\left.\Gamma_{0}\right) \backslash G$, let $R$ be the largest number $r$ with $\{|z|<r\} \subset \operatorname{int} \Gamma_{0}$. Then

$$
\left|f\left(z_{0}\right)\right| \leq 300 \mu\left(\left|z_{0}\right|\right) \quad \text { for }\left|z_{0}\right| \geq R, z_{0} \in \bar{G} .
$$


If $0<\eta<\frac{1}{8}$ and if $z_{0}$ is a point of $G$ with $\left|z_{0}\right|<R$ such that

$$
\operatorname{cap}\left(\left\{|z|<\frac{1}{2}\left|z_{0}\right|\right\} \cap(\mathbf{C} \backslash G)\right) \geq \eta\left|z_{0}\right|
$$

then

$$
\left|f\left(z_{0}\right)\right| \leq 27(2 \eta)^{-1} \mu\left(\left|z_{0}\right|\right)
$$

for this $z_{0}$.

If (7.3) is false for some $z_{0} \in G$ with $\left|z_{0}\right|<R$, set

$$
\rho=\sup \left\{\left|z_{0}\right|:\left|z_{0}\right|<R, z_{0} \in G \text { and (7.3) is false }\right\} \leq R .
$$

Then

$$
\left|f\left(z_{0}\right)\right| \leq 587 \eta^{-1} \mu\left(\left|z_{0}\right|\right) \quad \text { for } \frac{1}{2} \rho \leq\left|z_{0}\right| \leq \rho, z_{0} \in \bar{G},
$$

and there is $r \in\left(\frac{1}{2} \rho, \rho\right)$, say $r=R_{1}$, such that $\{|z|=r\} \subset G$. Furthermore, if $G_{1}=G \cap\left\{|z|<R_{1}\right\}$, then

$$
\operatorname{cap}\left(\left\{|z|<R_{1}\right\} \cap\left(\mathbf{C} \backslash G_{1}\right)\right) \leq 2 \eta R_{1} .
$$

We postpone the proof of Lemma 4 to $\S 8$.

7.2. To prove Theorem 4 , we may assume in view of Theorem 3 that $G$ is doubly connected and otherwise as in $\S 4$. By Lemma $\mathrm{A}$ it suffices to assume that $z_{1}=0 \notin G$ and $|f(z)| \leq \mu(|z|)$ for $z \in \partial G$. In view of Lemma 4 and (7.1), to prove that

$$
\left|f\left(z_{0}\right)\right| \leq 1.63 \cdot 10^{7} \mu\left(\left|z_{0}\right|\right)
$$

for $z_{0} \in G$, we may assume that $z_{1}=0 \in \Gamma_{1}$. For if $0 \in \Gamma_{0}$, then (7.8) follows from (7.1). We apply Lemma 4 to $G$ with $\eta=\frac{1}{16}$. It follows that there is nothing to prove unless there is $R_{1} \leq R$ as in Lemma 4 such that (7.7) holds. Then it remains to prove (7.8) when $\left|z_{0}\right|<R_{1}$. Note that $\Gamma_{1} \subset\left\{|z|<R_{1}\right\}$.

Now we have a function $f$ analytic in a neighborhood of $\bar{G}_{1}$ where $G_{1}=$ $G \cap\left\{|z|<R_{1}\right\}$ is doubly connected. We have, by (7.6),

$$
|f(z)| \leq \mu_{1}(|z|)=9392 \mu(|z|) \quad \text { for } z \in \partial G_{1} .
$$

Set $\rho_{0}=\max \left\{|z|: z \in \Gamma_{1}\right\}$. Then $\frac{\rho_{0}}{4} \leq \operatorname{cap} \Gamma_{1} \leq \frac{R_{1}}{8}$ by (7.7), so that $\rho_{0} \leq \frac{1}{2} R_{1}$. If $\left|z_{0}\right| \leq 2 \rho_{0}$ or $\left|z_{0}\right| \geq \frac{1}{2} R_{1}$, we can apply Theorem 1 to $G_{1}$ and $\mu_{1}$ with $w_{1}=0, w_{2}=z_{0}$ to obtain

$$
\left|f\left(z_{0}\right)\right| \leq 108 \mu_{1}\left(\left|z_{0}\right|\right)=1015000 \mu\left(\left|z_{0}\right|\right) .
$$

If $\left|z_{0}\right| \leq 2 \rho_{0}$, we take $\Omega=\left\{|z| \leq \frac{1}{2}\left|z_{0}\right|\right\}, A_{1}=\frac{3}{2}$ and $A_{2}=\frac{1}{8}$, so that $d \geq \frac{1}{2}\left|z_{0}\right|$. If $\left|z_{0}\right| \geq \frac{1}{2} R_{1}$, we take $\Omega=\left\{|z|=R_{1}, \operatorname{Re} \bar{z}_{0} z \leq 0\right\}, A_{1}=3$ and $A_{2}=\frac{1}{\sqrt{2}}$, so that $d \geq\left|z_{0}\right| \sqrt{2}$. 
7.3. Now consider $f$ in the annulus $G_{2}=\left\{\rho_{0}<|z|<R_{1}\right\} \subset G$. Then

$$
|f(z)| \leq \mu_{2}(|z|)=1015000 \mu(|z|) \quad \text { for } z \in \partial G_{2} .
$$

Recall that Lemma 1 is valid as long as $z_{1}=0 \notin G_{2}$ even if $0 \notin \partial G_{2}$.

Suppose that $2 \rho_{0}<|z|<\frac{1}{2} R_{1}$, so that $4 \rho_{0}<R_{1}$. We set $M(t)=$ $\log \mu_{2}\left(e^{t}\right)$. Let $m \geq 0$ be the integer with $2^{m-1}<\frac{\mu_{2}\left(R_{1}\right)}{\mu_{2}\left(4 \rho_{0}\right)} \leq 2^{m}$.

If $m=0$, then $\mu_{2}\left(R_{1}\right)=\mu_{2}\left(4 \rho_{0}\right)$, and since $|f(z)| \leq \mu_{2}\left(R_{1}\right)$ for $z \in \partial G_{2}$, the same holds for all $z \in G_{2}$ by the maximum principle. Hence $|f(z)| \leq$ $\mu_{2}\left(4 \rho_{0}\right) \leq 2 \mu_{2}(|z|)$ if $|z|>2 \rho_{0}$.

So we may assume that $m \geq 1$, and we set $t_{0}=\log R_{1}-m \log 2$. By (1.1), we have $M(t-\log 2) \geq M(t)-\log 2$ for all $t$. By repeated application of this we get

$$
M\left(t_{0}+\log 2\right) \geq M\left(\log R_{1}\right)-(m-1) \log 2>M\left(\log 4 \rho_{0}\right),
$$

so that $t_{0} \geq \log 2 \rho_{0}$ since $M(t)$ is nondecreasing. We define

$$
M_{1}(t)=\log \left(\mu_{2}\left(R_{1}\right) 2^{-m}\right) \geq \log \frac{1}{2} \mu_{2}\left(4 \rho_{0}\right) \quad \text { for } t \leq t_{0}
$$

and $M_{1}(t)=M\left(\log R_{1}\right)$ for $t \geq \log R_{1}$. We take $M_{1}(t)$ to be linear and continuous on $\left[t_{0}, \log R_{1}\right]$, so that the slope of $M_{1}$ is one on this interval and zero elsewhere.

By (1.1) we have $M(t+\log 2) \leq M(t)+\log 2$ for all real $t$. Therefore one can verify that

$$
M(t) \geq M_{1}(t)-\log 2
$$

for $\log \rho_{0} \leq t \leq \log R_{1}$. Furthermore,

$$
\log \frac{1}{2}|f(z)| \leq M_{1}(\log |z|) \quad \text { for } z \in \partial G_{2} .
$$

It follows that we can apply (6.7) and (6.8) to $G_{2}$ and $M_{1}(t)$ instead of $G$ and $M(t)$ with $j=0$, where $\Gamma_{0}$ now denotes the circle $\left\{|z|=R_{1}\right\}$. After a calculation involving the one-dimensional delta-distribution we get

$$
\log \frac{1}{2}\left|f\left(z_{0}\right)\right|-M_{1}\left(\log \left|z_{0}\right|\right) \leq \frac{1}{2 \pi} \int_{0}^{2 \pi} g\left(T e^{i \vartheta}\right) d \vartheta-h_{0}\left(\gamma_{0}\right)
$$

where

$$
\gamma_{0}=-1+\frac{1}{2 \pi} \int_{0}^{2 \pi} \omega_{0}\left(T e^{i \vartheta}\right) d \vartheta
$$

and $T=e^{t_{0}}$. Of course, $g(z)=g\left(z, z_{0}\right)$ is the Green's function of $G_{2}$ with the pole at $z=z_{0}$. 
7.4. Recall that we extend $g$ and $\omega_{0}$ to be zero in $\left\{|z|<R_{1}\right\} \backslash G_{2}$. We make some remarks that would apply also in a more general situation. The function $\omega_{0}$ is subharmonic in $\left\{|z|<R_{1}\right\}$ while $g$ is subharmonic in the disk $\left\{|z|<\left|z_{0}\right|\right\}$ and harmonic in the annulus $\left\{\left|z_{0}\right|<|z|<R_{1}\right\}$. It follows that the mean

$$
W(r)=\frac{1}{2 \pi} \int_{0}^{2 \pi} \omega_{0}\left(r e^{i \vartheta}\right) d \vartheta
$$

is an increasing convex function of $\log r$ for $0<r \leq\left|R_{1}\right|$ and linear in $\log r$ for $\rho_{0} \leq r \leq R_{1}$. The mean

$$
G(r)=\frac{1}{2 \pi} \int_{0}^{2 \pi} g\left(r e^{i \vartheta}\right) d \vartheta
$$

is an increasing convex function of $\log r$ for $0<r \leq\left|z_{0}\right|$ and a decreasing convex function of $\log r$ for $\left|z_{0}\right| \leq r \leq R_{1}$. Also $G(r)$ is linear in $\log r$ for $\rho_{0} \leq r \leq\left|z_{0}\right|$ and for $\left|z_{0}\right| \leq r \leq R_{1}$.

To prove (7.8), it suffices now, in view of (7.9), (7.10), (7.11) and (7.12), to show that

$$
G(r) \leq h_{0}(W(r))+2 \log 2
$$

for $2 \rho_{0} \leq r \leq R_{1}$, when $2 \rho_{0}<\left|z_{0}\right|<\frac{1}{2} R_{1}$. It follows from the properties of $G(r)$ and $W(r)$ above and from the concavity of $h_{0}$ on $[0,1]$ that $H(r)=$ $G(r)-h_{0}(W(r))$ is convex in $\log r$ for $\rho_{0} \leq r \leq\left|z_{0}\right|$ and for $\left|z_{0}\right| \leq r \leq R_{1}$ and thus attains its maximum when $r$ is equal to $\rho_{0}$ or $\left|z_{0}\right|$ or $R_{1}$. We have $H\left(\rho_{0}\right)=H\left(R_{1}\right)=0$.

We can write $g(z)=u(z)-\log \left|z-z_{0}\right|$, where $u$ is harmonic in $G_{2}$ with boundary values $\log \left|z-z_{0}\right|$. Hence $G(r)=U(r)-L(r)$ where

$$
U(r)=\frac{1}{2 \pi} \int_{0}^{2 \pi} u\left(r e^{i \vartheta}\right) d \vartheta
$$

is a linear function of $\log r$ for $\rho_{0} \leq r \leq R_{1}$ while

$$
L(r)=\frac{1}{2 \pi} \int_{0}^{2 \pi} \log \left|r e^{i \vartheta}-z_{0}\right| d \vartheta=\log \left|z_{0}\right|+\log ^{+}\left(\frac{r}{\left|z_{0}\right|}\right) .
$$

Since $G_{2}$ is the annulus $\left\{\rho_{0}<|z|<R_{1}\right\}$, we have

$$
\begin{aligned}
& U\left(\rho_{0}\right)=\log \left|z_{0}\right| \text { and } \\
& U\left(R_{1}\right)=\log R_{1} .
\end{aligned}
$$


Hence

$$
\begin{aligned}
G\left(\left|z_{0}\right|\right) & =\frac{\left(\log \frac{R_{1}}{\left|z_{0}\right|}\right) \log \left|z_{0}\right|+\left(\log \frac{\left|z_{0}\right|}{\rho_{0}}\right) \log R_{1}}{\log \left(\frac{R_{1}}{\rho_{0}}\right)}-\log \left|z_{0}\right| \\
& =\frac{\log \left(\frac{\left|z_{0}\right|}{\rho_{0}}\right)}{\log \left(\frac{R_{1}}{\rho_{0}}\right)} \log \left(\frac{R_{1}}{\left|z_{0}\right|}\right) .
\end{aligned}
$$

Further, since the level curves of

$$
\omega_{0}(z)=\frac{\log \frac{|z|}{\rho_{0}}}{\log \frac{R_{1}}{\rho_{0}}}
$$

are circles centered at the origin, we have

$$
h_{0}\left(W\left(\left|z_{0}\right|\right)\right)=g\left(-z_{0}\right)=u\left(-z_{0}\right)-\log 2\left|z_{0}\right| .
$$

If $v$ is the harmonic function in $G_{2}$ with $v(z)=\log \left(\left|z_{0}\right|-\rho_{0}\right)$ for $|z|=\rho_{0}$ and $v(z)=\log \left(R_{1}-\left|z_{0}\right|\right)$ for $|z|=R_{1}$, then $u \geq v$ on $\partial G_{2}$ and hence in $G_{2}$. Therefore

$$
\begin{aligned}
u\left(-z_{0}\right) \geq & v\left(-z_{0}\right) \\
& =\frac{\log \left(\frac{R_{1}}{\left|z_{0}\right|}\right)}{\log \left(\frac{R_{1}}{\rho_{0}}\right)} \log \left(\left|z_{0}\right|-\rho_{0}\right)+\frac{\log \left(\frac{\left|z_{0}\right|}{\rho_{0}}\right)}{\log \left(\frac{R_{1}}{\rho_{0}}\right)} \log \left(R_{1}-\left|z_{0}\right|\right) .
\end{aligned}
$$

One can verify that it follows from (7.14), (7.15), and (7.16) that

$$
G\left(\left|z_{0}\right|\right)-h_{0}\left(W\left(\left|z_{0}\right|\right)\right) \leq 2 \log 2
$$

since $2 \rho_{0} \leq\left|z_{0}\right| \leq \frac{1}{2} R_{1}$. Namely, we may normalize $\rho_{0}=1$ and set $\left|z_{0}\right|=r \in$ $\left(2, \frac{1}{2} R_{1}\right)$. Then

$$
\begin{aligned}
H(r) & =\log 2-\frac{\log \left(\frac{R_{1}}{r}\right)}{\log R_{1}} \log \left(1-r^{-1}\right)-\frac{\log r}{\log R_{1}} \log \left(1-\frac{r}{R_{1}}\right) \\
& \leq\left(3-\frac{\log \left(2 R_{1}-4\right)}{\log R_{1}}\right) \log 2 \leq 2 \log 2,
\end{aligned}
$$

since this function $H(r)$ attains its maximum at $r=2$ and at $r=\frac{R_{1}}{2}$. This proves (7.13) and Theorem 4 is proved. 
7.5. The proof of Theorem 4 above does not seem to have a straightforward generalization to domains of higher connectivity, at least if we only make use of $h_{0}$ but not of the other functions $h_{j}$. The most serious reason for this is that the level curves of $\omega_{0}(z)$ may get so close to $\partial G$ that the minimum of $g\left(z, z_{0}\right)$ remains small on them. Then the term $-h_{0}\left(\gamma_{0}\right)$ is not of much help in (6.7).

The following example is due to Jang-Mei Wu. Let $G$ be the triply connected domain with boundary curves $\Gamma_{0}=\{|z|=R\}, \Gamma_{1}=\{|z-\varepsilon|=\varepsilon\}$ and $\Gamma_{2}=$ $\{|z-b|=\delta\}$ where $R$ is large, $\varepsilon$ and $\delta$ are small, $1<b<R$ and $R-b$ is small. Take $z_{0}=1 \in G$. The level curve $\left\{\omega_{0}(z)=t\right\}$ passes through the narrow part between $\Gamma_{0}$ and $\Gamma_{2}$ for any $t$, and $h(t)<1$ for all $t$ if $\delta$ and $R-b$ are small enough. If $M(t)=0$ for $t \leq 0$ and $M(t)=t$ for $t>0$, then the right-hand side of (6.7) is not smaller than

$$
I=\frac{1}{2 \pi} \int_{0}^{2 \pi} g\left(e^{i \vartheta}, 1\right) d \vartheta-1
$$

By taking $\varepsilon$ small enough, one can achieve $I \geq c \log R$ for some positive constant $c$, so that the right-hand side of (6.7) cannot remain below an absolute constant in this case. One can ask what happens if we use (6.7) with $j=1$ or $j=2$. On the other hand, in this particular case, one could use here Theorem 1 first to prove (1.3) for $z_{1}=0$ and all $z_{2}$ on a set joining $\Gamma_{0}$ and $\Gamma_{2}$ and then use other ideas in a smaller domain obtained by joining $\Gamma_{0}$ and $\Gamma_{2}$.

More generally, given a domain $G$ with $0 \in \partial G$, and a function $f$ with $|f(z)| \leq \mu(|z|)$ for $z \in \partial G$, one could first try to use Theorem 1 to show that there is a subdomain $G_{1}$ such that the components of $\partial G_{1}$ are well separated and $|f(z)| \leq C \mu(|z|)$ for $z \in \partial G_{1}$, where $C$ might depend on the connectivity $N+1$ of $G$. After that we use Theorem 1 to estimate $\left|f\left(z_{0}\right)\right|$ if $z_{0} \in G_{1}$ is close to some component of $\partial G_{1}$, and try to use the Teichmüller method otherwise. By well-separated we mean that the diameter of a component $\Gamma$ of $\partial G_{1}$ should not exceed an absolute constant times the distance of $\Gamma$ from $\partial G_{1} \backslash \Gamma$. Also, the connectivity of $G_{1}$ is not to exceed that of $G$.

Instead of considering the harmonic measures $\omega_{i}$ of the individual boundary components $\Gamma_{i}$, we could pool some curves $\Gamma_{i}$ together, denote the sum of their harmonic measures by $\omega(z)$, and then define a function $h$ by means of (6.1), replacing $\omega_{j}$ by $\omega$ in (6.1). If $z_{0}$ is given and we group together those $\Gamma_{i}$ which, in some sense, are closest to $z_{0}$, we might obtain a situation that to a greater extent resembles that of an annulus. Of course to make this suggestion due to $\mathrm{Wu}$ precise and to make it work, if possible, quite a few technical details would have to be taken care of. 


\section{$\S 8$. Proof of Lemma 4}

8.1. Let $G$ and $f$ be as in Lemma 4. Suppose that $z_{1}=0 \in \Gamma_{0}$. The same argument that we used to deduce Theorem $\mathrm{A}$ from Theorem 1 in subsection 2.2 shows that $\left|f\left(z_{0}\right)\right| \leq 74 \mu\left(\left|z_{0}\right|\right)$ for $z_{0} \in G$. Suppose then that $0 \in\left(\operatorname{int} \Gamma_{0}\right) \backslash G$ and let $R$ be as in Lemma 4. If $z_{0} \in G$ and $\left|z_{0}\right| \geq R$, we apply Theorem 1 to $w_{1}=0$ and $w_{2}=z_{0}$. We set $B=\left\{\left|z-z_{0}\right|<\frac{1}{2}\left|z_{0}\right|\right\}$. We cannot have $\Gamma_{0} \subset \bar{B}$. So $\Gamma_{0}$ intersects $\partial B$ or $\Gamma_{0}$ contains a point in $\{|z|=R\} \backslash \bar{B}$. In each case one can find a closed arc of $\Gamma_{0}$ of diameter $\frac{1}{2}\left|z_{0}\right|$ remaining outside $B$. In the first case, the arc starts at a point on $\partial B$ and in the second case at a point on $\{|z|=R\}$. We take $\Omega$ to be such an arc. Then we can take $A_{1}=\frac{5}{2}$ and $A_{2}=\frac{1}{8}$ in Theorem 1 , while $d \geq \frac{1}{2}\left|z_{0}\right|$. This gives (7.2).

Suppose next that $0<\eta<\frac{1}{8},\left|z_{0}\right|<R$ and that (7.3) holds. We apply Theorem 1 with $\Omega=\left\{|z| \leq \frac{1}{2}\left|z_{0}\right|\right\}, A_{1}=\frac{3}{2}$ and $A_{2}=\eta$, and obtain (7.4).

Let $\rho$ be given by (7.5). If $\frac{1}{2} \rho \leq\left|z_{0}\right|<\rho$, we apply Theorem 1 to the open set $G_{1}=G \cap\{|z|<\rho\}$ and to $27(2 \eta)^{-1} \mu$ instead of $G$ and $\mu$, with $w_{1}=$ $0, w_{2}=z_{0}, \Omega=\left\{z:|z|=\rho\right.$ and $\left.\operatorname{Re} \bar{z}_{0} z \leq 0\right\}, A_{1}=3$ and $A_{2}=\frac{1}{\sqrt{2}}$ (cf. [1, Theorem 2.7, p. 35]). Since $d \geq\left|z_{0}\right| \sqrt{2}$, we get (7.6).

Next we show that there is $r \in\left(\frac{1}{2} \rho, \rho\right)$ such that $S(r)=\{|z|=r\} \subset G$. For suppose that this is not the case. Set $F=\partial G \cap\left\{\frac{1}{2} \rho<|z|<\rho\right\}$. Then $\operatorname{cap} F \leq$ $\eta \rho$. Let $F_{1}$ be the circular projection of $F$ onto the positive real axis. Then cap $F_{1} \leq \operatorname{cap} F$, while $F_{1} \supset\left(\frac{1}{2} \rho, \rho\right)$ by our assumption and hence cap $F_{1} \geq \frac{\rho}{8}$. This gives $\eta \geq \frac{1}{8}$, which is a contradiction. So $S(r) \subset G$ for some $r \in\left(\frac{1}{2} \rho, \rho\right)$, which we denote by $R_{1}$.

If $G_{1}=G \cap\left\{|z|<R_{1}\right\}$, then $\partial G_{1}$ consists of $S\left(R_{1}\right)$ together with at most $N$ of the analytic curves $\Gamma_{i}$, which lie completely in $\left\{|z|<R_{1}\right\}$. Also

$$
\operatorname{cap}\left(\left(\mathbf{C} \backslash G_{1}\right) \cap\left\{|z|<R_{1}\right\}\right) \leq \operatorname{cap}((\mathbf{C} \backslash G) \cap\{|z|<\rho\}) \leq \eta \rho \leq(2 \eta) R_{1},
$$

which gives (7.7). Lemma 4 is proved.

8.2. We may assume that $R_{1}=1$ in Lemma 4 . Hence in view of Theorems 2 and 3 and Lemma 4 the remaining open problem can be formulated as follows.

Problem. Let $G$ be a domain obtained by deleting from the unit disk $\Delta, N$ disjoint analytic Jordan curves that lie in $\Delta$, and their interiors. Let $f$ be analytic in a neighborhood of $\bar{G}$, and suppose that $0 \in \partial G, f(0)=0$, and $|f(z)| \leq \mu(|z|)$ for all $z \in \partial G$. Suppose further that the capacity of $\mathbf{C} \backslash G$ in $\Delta$ does not exceed a positive absolute constant $\eta$. Is it true that then $|f(z)| \leq$ $C \mu(|z|)$ for all $z \in G$ where $C$ is a constant depending at most on $N$ ?

An affirmative answer to this problem would show that the assumptions and hence the conclusions of Theorems 2 or 3 are satisfied, depending on whether or not $C$ depends on $N$. 


\section{$\S 9$. When $G$ is not connected}

9.1. Let $G$ be an open set with at least two components, each of which is bounded. Let $f$ be analytic in $G$ and continuous in $\bar{G}$, and suppose that (1.2) holds for a fixed $z_{1} \in \partial G$ and all $z_{2} \in \partial G$. Let $D_{1}$ be a component of $G$ such that $z_{1} \in \partial D_{1}$ (such a component can be found, for example, if $G$ has only finitely many components, as in Theorem 2). Suppose that $z_{0} \in D_{2}$, where $D_{2}$ is a component of $G$ other than $D_{1}$, and that $z_{1} \in D_{3}$, the unbounded component of the complement of $\bar{D}_{2}$.

Lemma 5. Under the assumptions above, we have

$$
\left|f\left(z_{0}\right)-f\left(z_{1}\right)\right| \leq C \mu\left(\left|z_{0}-z_{1}\right|\right)
$$

where $C=217$.

We set $d_{1}=\left|z_{0}-z_{1}\right|$ and $\Gamma_{0}=\partial D_{3}$. Let $z_{3} \in \Gamma_{0}$ be a point on the line segment joining $z_{1}$ to $z_{0}$, and set $d_{2}=\left|z_{1}-z_{3}\right|$. Then $d_{1}=d_{2}+\left|z_{0}-z_{3}\right|$. Set $A=\mu\left(d_{2}\right) \leq \mu\left(d_{1}\right)$. Then for any $z \in \partial D_{2}$ we have

$$
\begin{aligned}
\left|f(z)-f\left(z_{3}\right)\right| & \leq\left|f(z)-f\left(z_{1}\right)\right|+\left|f\left(z_{1}\right)-f\left(z_{3}\right)\right| \\
& \leq \mu\left(\left|z-z_{1}\right|\right)+A \\
& \leq \mu\left(\left|z-z_{3}\right|+d_{2}\right)+A \\
& =\nu\left(\left|z-z_{3}\right|\right),
\end{aligned}
$$

say, where $\nu(t)$ satisfies (1.1) since $\mu$ does. Since $z_{3} \in \Gamma_{0}$, we can apply Theorem 1 to $D_{2}$ and $\nu$ instead of $G$ and $\mu$, with $w_{1}=z_{3}, w_{2}=z_{0}$,

$$
\Omega=\left\{\left|z-z_{3}\right| \leq \frac{1}{2}\left|z_{0}-z_{3}\right|\right\}
$$

$A_{1}=\frac{3}{2}$ and $A_{2}=\frac{1}{8}$, noting that $d \geq d\left(z_{0}, \Omega\right)=\frac{1}{2}\left|z_{0}-z_{3}\right|$. This gives

$$
\begin{aligned}
\left|f\left(z_{0}\right)-f\left(z_{3}\right)\right| & \leq 108 \nu\left(\left|z_{0}-z_{3}\right|\right) \\
& =108 \mu\left(\left|z_{0}-z_{3}\right|+d_{2}\right)+108 A \\
& \leq 216 \mu\left(d_{1}\right) .
\end{aligned}
$$

Hence

$$
\left|f\left(z_{0}\right)-f\left(z_{1}\right)\right| \leq 216 \mu\left(\left|z_{0}-z_{1}\right|\right)+\mu\left(\left|z_{1}-z_{3}\right|\right) \leq 217 \mu\left(d_{1}\right),
$$

and (9.1) holds with $C=217$, as asserted.

9.2. We remark that if $z_{1}$ belongs to one of the bounded components of the complement of $D_{2}$, then the problem of estimating $\left|f\left(z_{0}\right)-f\left(z_{1}\right)\right|$ is essentially the same as the problem formulated in subsection 8.2. 


\section{References}

[1] L. V. Ahlfors, Conformal Invariants, McGraw-Hill, New York, 1973.

[2] J. Garnet , Analytic capacity and measure, Lecture Notes in Math. 297 Springer, New York, 1972.

[3] F. W. Gehring, W. K. Hayman \& A. Hinkkanen, Analytic functions satisfying Hölder conditions on the boundary, J. Approx. Theory 35 (1982), 243-249.

[4] G. H. Hardy \& J. E. Littlewood, Some properties of fractional integrals II, Math. Z. 34 (1931), 403-439.

[5] A. Hinkkanen, On the modulus of continuity of analytic functions, Ann. Acad. Sci. Fenn. Series A1 10 (1985), 247-253.

[6] L. A. Rubel, A. L. Shields \& B. A. TAylor, Mergelyan sets and the modulus of continuity of analytic functions, J. Approx. Theory 15 (1975), 23-40.

[7] W. Rudin, Real and Complex Analysis, McGraw-Hill, Hightstown, New Jersey, 1966 .

[8] W. E. Sewell, Degree of Approximation by Polynomials in the Complex Domain, Princeton Univ. Press, Princeton, New Jersey, 1942.

[9] P. M. TAMrazov, Contour and solid structure properties of holomorphic functions of a complex variable, Russian Math. Surveys 28 (1973), 141-173.

[10] P. M. TAMrAzov, Holomorphic functions and mappings in the contour-solid problem, Akad. Nauk. SSSR Dokl. 279 (1984), 38-43; English transl. in Soviet Math. Dokl. 30 (1984), 606-611.

[11] O. Teichmüller, Eine Verschärfung des Dreikreisesatzes, Deutsche Math. 4 (1939), 16-22; also pp. 276-282 in "Collected Papers".

[12] O. Teichmüller, Collected Papers, (L. V. Ahlfors \& F. W. Gehring, Eds.), Springer, Berlin/Heidelberg/New York, 1982.

[13] M. Tsuji, Potential Theory in Modern Function Theory, Maruzen, Tokyo, 1959.

[14] J. L. WAlsh \& W. E. SeWELl, Sufficient conditions for various degrees of approximation by polynomials, Duke Math. J. 6 (1940), 658-705.

This work was partially supported by a grant from the National Science Foundation.

Department of Mathematics

University of Michigan

Ann Arbor, Michigan 48109-1003

Received December 23, 1985. 